\title{
Measure and Detection of Genuine Multipartite Entanglement for Tripartite Systems
}

\author{
Ming $\mathrm{Li}^{1}$, Lingxia Jia ${ }^{1}$, Jing Wang ${ }^{1}$, Shuqian Shen ${ }^{1}$, and Shao-Ming $\mathrm{Fi}^{2}{ }^{2,3}$ \\ ${ }^{1}$ College of the Science, China University of Petroleum, 266580 Qingdao, China \\ ${ }^{2}$ School of Mathematical Sciences, Capital Normal University, Beijing 100048, China \\ 3 Max-Planck-Institute for Mathematics in the Sciences, 04103 Leipzig, Germany
}

\begin{abstract}
It is a computationally hard task to certify genuine multipartite entanglement (GME). We investigate the relation between the norms of the correlation vectors and the detection of GME for tripartite quantum systems. A sufficient condition for GME and an effective lower bound for the GME concurrence are derived. Several examples are considered to show the effectiveness of the criterion and the lower bound of GME concurrence.
\end{abstract}

PACS numbers: 03.67.-a, 02.20.Hj, 03.65.-w

\section{INTRODUCTION}

Quantum entanglement is a remarkable resource in the theory of quantum information, with various applications [1, 2]. A multipartite quantum state that is not separable with respect to any bi-partition is said to be genuine multipartite entangled [3]. As one of the important type of entanglement, genuine multipartite entanglement(GME) offers significant advantage in quantum tasks comparing with bipartite entanglement [4]. In particular, it is the basic ingredient in measurement-based quantum computation [5], and is beneficial in various quantum communication protocols [6], including secret sharing [7] (cf. [8]), extreme spin squeezing [9], high sensitivity in some general metrology tasks [10], quantum computing using cluster states [11], and multiparty quantum network $13-$ 15]. However, detecting and measuring quantum entanglement turn out to be quite difficult. To detect GME, series of linear and nonlinear entanglement witnesses 1724, 26, 27], generalized concurrence for GME 28 31], and Bell-like inequalities [32] were derived and a characterization in terms of semi-definite programs (SDP) was developed [33]. Nevertheless, the problem remains far from being satisfactorily solved.

From the norms of the correlation tensors in the generalized Bloch representation of a quantum state, separable conditions for both bi- and multi-partite quantum states have been presented in 38 41]. A multipartite entanglement measure for $\mathrm{N}$-qubit and $\mathrm{N}$-qudit pure states is given in [42, 43]. A general framework for detecting genuine multipartite entanglement and non full separability in multipartite quantum systems of arbitrary dimensions has been introduced in [19]. In [44] it has been shown that the norms of the correlation tensors has a close relationship to the maximal violation of a kind of multi Bell inequalities and to the concurrence [45].

In this paper, we investigate the genuine tripartite entanglement in terms of the norms of the correlation tensors and GME concurrence for tripartite qudit quantum systems. We derive criteria to detect GME. An effective lower bound for GME concurrence is also presented.

\section{CRITERIA FOR DETECTING GME}

In this section, we present a criterion to detect GME by using the approach presented in [19] for tripartite qudit systems. We start with some definitions and notations.

Let $H_{i}^{d}, i=1,2,3$, denote $d$-dimensional Hilbert spaces. A tripartite state $\rho \in H_{1}^{d} \otimes H_{2}^{d} \otimes H_{3}^{d}$ can be expressed as $\rho=\sum p_{\alpha}\left|\psi_{\alpha}\right\rangle\left\langle\psi_{\alpha}\right|$, where $0<p_{\alpha} \leq 1$, $\sum p_{\alpha}=1,\left|\psi_{\alpha}\right\rangle \in H_{1}^{d} \otimes H_{2}^{d} \otimes H_{3}^{d}$ are normalized pure states. If all $\left|\psi_{\alpha}\right\rangle$ are biseparable, namely, either $\left|\psi_{\alpha}\right\rangle=\left|\varphi_{\alpha}^{1}\right\rangle \otimes\left|\varphi_{\alpha}^{23}\right\rangle$ or $\left|\psi_{\beta}\right\rangle=\left|\varphi_{\beta}^{2}\right\rangle \otimes\left|\varphi_{\beta}^{13}\right\rangle$ or $\left|\psi_{\gamma}\right\rangle=\left|\varphi_{\gamma}^{3}\right\rangle \otimes\left|\varphi_{\gamma}^{12}\right\rangle$, where $\left|\varphi_{\gamma}^{i}\right\rangle$ and $\left|\varphi_{\gamma}^{i j}\right\rangle$ denote pure states in $H_{i}^{d}$ and $H_{i}^{d} \otimes H_{j}^{d}$ respectively, then $\rho$ is said to be bipartite separable. Otherwise, $\rho$ is called genuine multipartite entangled.

Let $\lambda_{i}, i=1, \cdots, d^{2}-1$, denote the generators of the special unitary group $S U(d)$ [46], and $I$ the $d \times d$ identity matrix . Any $\rho \in H_{1}^{d} \otimes H_{2}^{d} \otimes H_{3}^{d}$ can be represented as follows:

$$
\begin{aligned}
\rho= & \frac{1}{d^{3}} I \otimes I \otimes I+\frac{1}{2 d^{2}}\left(\sum t_{i}^{1} \lambda_{i} \otimes I \otimes I+\sum t_{j}^{2} I \otimes \lambda_{j} \otimes I+\sum t_{k}^{3} I \otimes I \otimes \lambda_{k}\right) \\
& +\frac{1}{4 d}\left(\sum t_{i j}^{12} \lambda_{i} \otimes \lambda_{j} \otimes I+\sum t_{i k}^{13} \lambda_{i} \otimes I \otimes \lambda_{k}+\sum t_{j k}^{23} I \otimes \lambda_{j} \otimes \lambda_{k}\right)+\frac{1}{8} \sum t_{i j k}^{123} \lambda_{i} \otimes \lambda_{j} \otimes \lambda_{k},
\end{aligned}
$$

where $t_{i}^{1}=\operatorname{tr}\left(\rho \lambda_{i} \otimes I \otimes I\right), t_{j}^{2}=\operatorname{tr}\left(\rho I \otimes \lambda_{j} \otimes I\right), \quad t_{k}^{3}=\operatorname{tr}\left(\rho I \otimes I \otimes \lambda_{k}\right), t_{i j}^{12}=\operatorname{tr}\left(\rho \lambda_{i} \otimes \lambda_{j} \otimes I\right) . \quad t_{i k}^{13}=$ 
$\operatorname{tr}\left(\rho \lambda_{i} \otimes I \otimes \lambda_{k}\right), t_{j k}^{23}=\operatorname{tr}\left(\rho I \otimes \lambda_{j} \otimes \lambda_{k}\right)$ and $t_{i j k}^{123}=$ $\operatorname{tr}\left(\rho \lambda_{i} \otimes \lambda_{j} \otimes \lambda_{k}\right)$. In the following, we set $T^{(1)}, T^{(2)}$, $T^{(3)}, T^{(12)}, T^{(13)}, T^{(23)}$ and $T^{(123)}$ to be the vectors (tensors) with entries $t_{i}^{1}, t_{j}^{2}, t_{k}^{3}, t_{i j}^{12}, t_{i k}^{13}, t_{j k}^{23}$ and $t_{i j k}^{123}$, $i, j, k=1,2, \cdots, d^{2}-1$, which are the so called correlation vectors.

Let $\|M\|_{k}=\sum_{i=1}^{k} \sigma_{i}$ denote the $k$-norm for an $n \times n$ matrix $M$, where $\sigma_{i}, i=1, \ldots, n$, are the singular values of $M$ in decreasing order. $\|M\|_{n}=\|M\|_{K F}$ is just the Key-Fan norm. Denote $\|\cdot\|$ the Frobenius norm of a vector or a matrix. Let $T_{\underline{12} 3}, T_{\underline{2} 13}$ and $T_{\underline{3} 12}$ be the matrices with entries $t_{i, d(j-1)+k}=t_{i j k}, t_{j, d(i-1)+k}=t_{i j k}$ and $t_{k, d(i-1)+j}=t_{i j k}$, respectively. Set $M_{k}(\rho)=\frac{1}{3}\left(\left\|T_{123}\right\|_{k}+\right.$ $\left.\left\|T_{\underline{2} 13}\right\|_{k}+\left\|T_{\underline{3} 12}\right\|_{k}\right)$.

Lemma: For a pure tripartite qudit state, we have for any $k=1,2, \cdots, d^{2}-1$ and $\underline{j l m}=\underline{123}, \underline{2} 13, \underline{3} 12$ that

(i) if the state is fully separable, then

$$
\left\|T_{\underline{j} l m}\right\|_{k} \leq \sqrt{\frac{8(d-1)^{3}}{d^{3}}}
$$

(ii) if the state is separable under bipartite partition $j \mid l m$, then

$$
\left\|T_{\underline{j} l m}\right\|_{k} \leq \sqrt{\frac{8(d-1)^{2}(d+1)}{d^{3}}}
$$

(iii) if the state is entangled under bipartite partition $j \mid l m$, then

$$
\left\|T_{\underline{j} l m}\right\|_{k} \leq \sqrt{\frac{8 k(d-1)^{2}(d+1)}{d^{3}}}
$$

Proof. We shall use repeatedly $\left\|T^{(i)}\right\| \leq \sqrt{\frac{2(d-1)}{d}}, i=$
$1,2,3,\left\|T^{(l m)}\right\| \leq 2 \sqrt{\frac{d^{2}-1}{d^{2}}}, l m=12,13,23$ [19] and $\|M\|_{k} \leq \sqrt{k}\|M\|$ for any matrix $M$. Then we have (i)

$$
\begin{aligned}
\left\|T_{\underline{j} l m}\right\|_{k} & =\left\|\left(T^{(j)}\right) \cdot\left(T^{(l)} \otimes T^{(m)}\right)^{t}\right\|_{k} \\
& =\left\|T^{(j)}\right\|\left\|T^{(l)} \otimes T^{(m)}\right\|=\left\|T^{(j)}\right\|\left\|T^{(l)}\right\|\left\|T^{(m)}\right\| \\
& \leq \sqrt{\frac{8(d-1)^{3}}{d^{3}}}
\end{aligned}
$$

(ii)

$$
\begin{aligned}
\left\|T_{\underline{j} l m}\right\|_{k} & =\left\|\left(T^{(j)}\right) \cdot\left(T^{(l m)}\right)^{t}\right\|_{k} \\
& =\left\|T^{(j)}\right\|\left\|T^{(l m)}\right\| \leq \sqrt{\frac{8(d-1)^{2}(d+1)}{d^{3}}}
\end{aligned}
$$

$$
\begin{aligned}
\left\|T_{\underline{j} l m}\right\|_{k} & =\left\|\left(T^{(\underline{j} l)}\right) \otimes\left(T^{(m)}\right)^{t}\right\|_{k} \\
& =\left\|T^{(\underline{j} l)}\right\|_{k}\left\|T^{(m)}\right\| \leq \sqrt{\frac{8 k(d-1)^{2}(d+1)}{d^{3}}} ;
\end{aligned}
$$

where we have denoted $T^{(\underline{j})}$ the matrix with entries $t_{x y}^{j l}$.

Theorem 1: If for a tripartite qudit state $\rho$, it holds that

$$
M_{k}(\rho)>\frac{2 \sqrt{2}}{3}(2 \sqrt{k}+1) \frac{d-1}{d} \sqrt{\frac{d+1}{d}}
$$

for any $k=1,2, \cdots, d^{2}-1$, then $\rho$ is genuine multipartite entangled.

Proof. Assume that $\rho$ is bipartite separable. By using the above Lemma, we get

$$
\begin{aligned}
M_{k}(\rho) & =\frac{1}{3}\left(\left\|T_{\underline{123}}(\rho)\right\|_{k}+\left\|T_{\underline{2} 13}(\rho)\right\|_{k}+\left\|T_{\underline{3} 12}(\rho)\right\|_{k}\right) \\
& =\frac{1}{3}\left(\| \sum_{\alpha} p_{\alpha} T_{\underline{1} 23}\left(\left|\psi_{\alpha}\right\rangle\right)\left\|_{k}+\right\| \sum_{\alpha} p_{\alpha} T_{\underline{2} 13}\left(\left|\psi_{\alpha}\right\rangle\right)\left\|_{k}+\right\| \sum_{\alpha} p_{\alpha} T_{\underline{3} 12}\left(\left|\psi_{\alpha}\right\rangle\right) \|_{k}\right) \\
& \leq \frac{1}{3} \sum_{\alpha} p_{\alpha}\left(\| T_{\underline{123}}\left(\left|\psi_{\alpha}\right\rangle\right)\left\|_{k}+\right\| T_{\underline{2} 13}\left(\left|\psi_{\alpha}\right\rangle\right)\left\|_{k}+\right\| T_{\underline{3} 12}\left(\left|\psi_{\alpha}\right\rangle\right) \|_{k}\right) \\
& \leq \frac{1}{3} \sum_{\alpha} p_{\alpha}\left(2 \sqrt{\frac{8 k(d-1)^{2}(d+1)}{d^{3}}}+\sqrt{\frac{8(d-1)^{2}(d+1)}{d^{3}}}\right) \\
& =\frac{1}{3}\left(2 \sqrt{\left.\frac{8 k(d-1)^{2}(d+1)}{d^{3}}+\sqrt{\frac{8(d-1)^{2}(d+1)}{d^{3}}}\right)}\right. \\
& =\frac{2 \sqrt{2}}{3}(2 \sqrt{k}+1) \frac{d-1}{d} \sqrt{\frac{d+1}{d}} .
\end{aligned}
$$


Thus once the inequality is violated, the quantum state $\rho$ must be genuine multipartite entangled.

Remark. For $d=2, k=3$, Theorem 1 reduces to the Theorem 2 in [19]. If we set $d=2$, and $k=1,2$ one finds that Theorem 1 is strictly covered by Theorem 2 in [19]. However, our theorem can detect GME for any tripartite qudit systems rather than only tripartite qubit systems.

Example 1: Consider quantum state $\rho \in H_{1}^{3} \otimes H_{2}^{3} \otimes$ $H_{3}^{3}, \rho=\frac{1-x}{27} I+x|\varphi\rangle\langle\varphi|$, where $|\varphi\rangle=\frac{1}{\sqrt{3}}(|000\rangle+|111\rangle+$ $|222\rangle)$ is the GHZ state. By Theorem 1 in [19] we can detect GME for $0.894427<x \leq 1$. With our Theorem 1 , by setting $d=3$ and $k=8$ (which is the optimal selection) one detects GME for $0.716235<x \leq 1$ (see Fig. 1).

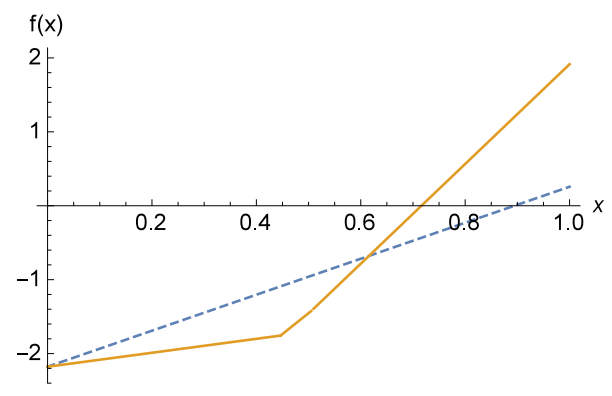

FIG. 1: Vicente criterion (dashed line) v.s. the lower bound (5) in this manuscript (solid line). We have used $f(x)$ to denote the difference between the left and the right side of the two criteria. From the figure one sees that $\rho$ is genuine multipartite entangled for $0.894427<x \leq 1$ by Vicente's result with $f(x)=-2.177324+2.434322 x$. One computes $f(x)=-4.83138+6.74552 x$ by our proposition. Thus GME is detected for $0.716235<x \leq 1$.

\section{LOWER BOUND OF GME CONCURRENCE}

The GME concurrence is proved a well defined measure [28, 29]. For a pure state $|\psi\rangle \in H_{1}^{d} \otimes H_{2}^{d} \otimes H_{3}^{d}$, the GME concurrence is defined by

$C_{G M E}(|\psi\rangle)=\sqrt{\min \left\{1-\operatorname{tr}\left(\rho_{1}^{2}\right), 1-\operatorname{tr}\left(\rho_{2}^{2}\right), 1-\operatorname{tr}\left(\rho_{3}^{2}\right)\right\}}$

where $\rho_{i}$ is the reduced matrix for the $i$ th subsystem. For mixed state $\rho \in H_{1}^{d} \otimes H_{2}^{d} \otimes H_{3}^{d}$, the GME concurrence is then defined by the convex roof

$$
C_{G M E}(\rho)=\min \sum_{\left\{p_{\alpha},\left|\psi_{\alpha}\right\rangle\right\}} p_{\alpha} C_{G M E}\left(\left|\psi_{\alpha}\right\rangle\right)
$$

The minimum is taken over all pure ensemble decompositions of $\rho$.

Since one has to find the optimal ensemble to carry out the minimization, the GME concurrence is hard to compute. In this section we derive an effective lower bound for GME concurrence in terms of the norms of the correlation tensors.

Theorem 2: For a tripartite qudit state $\rho$, the GME concurrence satisfies the following inequality,

$$
C_{G M E}(\rho) \geq \max \left\{\frac{1}{2 \sqrt{2}}\left\|T^{(123)}\right\|-\frac{(d-1)}{d}, 0\right\} .
$$

Proof. We first consider pure states. For pure state $\rho=|\psi\rangle\langle\psi|$ one has $\operatorname{tr} \rho^{2}=1$, which implies that

$$
\operatorname{tr} \rho^{2}=\frac{1}{d^{3}}+\frac{1}{2 d^{2}} \sum\left(t_{i}^{1}\right)^{2}+\sum\left(t_{j}^{2}\right)^{2}+\sum\left(t_{k}^{3}\right)^{2}+\frac{1}{4 d} \sum\left(t_{i j}^{12}\right)^{2}+\sum\left(t_{i k}^{13}\right)^{2}+\sum\left(t_{j k}^{23}\right)^{2}+\frac{1}{8} \sum\left(t_{i j k}^{123}\right)^{2}=1 .
$$

From (8) we have

$$
\frac{1}{d^{3}}+\frac{1}{2 d^{2}}\left(\left\|T^{(1)}\right\|^{2}+\left\|T^{(2)}\right\|^{2}+\left\|T^{(3)}\right\|^{2}\right)+\frac{1}{4 d}\left(\left\|T^{(12)}\right\|^{2}+\left\|T^{(13)}\right\|^{2}+\left\|T^{(23)}\right\|^{2}\right)+\frac{1}{8}\left\|T^{(123)}\right\|^{2}=1 .
$$

In the following we denote $\rho_{j k}$ the reduced density matrix for the subsystems $j \neq k=1,2,3$. One computes from (11) that

$$
\rho_{1}=\frac{1}{d} I+\frac{1}{2} \sum t_{i}^{1} \lambda_{i}^{1}, \quad \rho_{23}=\frac{1}{d} I \otimes I+\frac{1}{2 d}\left(\sum t_{j}^{2} \lambda_{j}^{2} \otimes I+\sum t_{k}^{3} I \otimes \lambda_{k}^{3}\right)+\frac{1}{4} \sum t_{j k}^{23} \lambda_{j} \otimes \lambda_{k} .
$$

Thus

$$
\operatorname{tr} \rho_{1}^{2}=\frac{1}{d}+\frac{1}{2}\left\|T^{(1)}\right\|^{2}, \quad \operatorname{tr} \rho_{23}^{2}=\frac{1}{d^{2}}+\frac{1}{2 d}\left(\left\|T^{(2)}\right\|^{2}+\left\|T^{(3)}\right\|^{2}\right)+\frac{1}{4}\left\|T^{(23)}\right\|^{2} .
$$

Similarly we get

$$
\begin{aligned}
& \operatorname{tr} \rho_{2}^{2}=\frac{1}{d}+\frac{1}{2}\left\|T^{(2)}\right\|^{2}, \quad \operatorname{tr} \rho_{13}^{2}=\frac{1}{d^{2}}+\frac{1}{2 d}\left(\left\|T^{(1)}\right\|^{2}+\left\|T^{(3)}\right\|^{2}\right)+\frac{1}{4}\left\|T^{(13)}\right\|^{2}, \\
& \operatorname{tr} \rho_{3}^{2}=\frac{1}{d}+\frac{1}{2}\left\|T^{(3)}\right\|^{2}, \quad \operatorname{tr} \rho_{12}^{2}=\frac{1}{d^{2}}+\frac{1}{2 d}\left(\left\|T^{(1)}\right\|^{2}+\left\|T^{(2)}\right\|^{2}\right)+\frac{1}{4}\left\|T^{(12)}\right\|^{2}
\end{aligned}
$$


By noticing that $\rho=|\psi\rangle\langle\psi|$ is a pure state, we get $\operatorname{tr} \rho_{i}^{2}=\operatorname{tr} \rho_{j k}^{2}$ for $i \neq j \neq k, i, j, k=1,2,3$. Then we have:

$\frac{3}{d}+\frac{1}{2}\left(\left\|T^{(1)}\right\|^{2}+\left\|T^{(2)}\right\|^{2}+\left\|T^{(3)}\right\|^{2}\right)=\frac{3}{d^{2}}+\frac{1}{d}\left(\left\|T^{(1)}\right\|^{2}+\left\|T^{(2)}\right\|^{2}+\left\|T^{(3)}\right\|^{2}\right)+\frac{1}{4}\left(\left\|T^{(12)}\right\|^{2}+\left\|T^{(13)}\right\|^{2}+\left\|T^{(23)}\right\|^{2}\right)$.

Set $A=\left\|T^{(1)}\right\|^{2}+\left\|T^{(2)}\right\|^{2}+\left\|T^{(3)}\right\|^{2}, B=\left\|T^{(12)}\right\|^{2}+\left\|T^{(13)}\right\|^{2}+\left\|T^{(23)}\right\|^{2}$ and $C=\left\|T^{(123)}\right\|^{2}$. We have

$$
\frac{1}{4} B=\left(\frac{1}{2}-\frac{1}{d}\right) A+\frac{3}{d}-\frac{2}{d^{2}} \text {. }
$$

Substituting (9) into (8), one has

$$
\begin{aligned}
\operatorname{tr} \rho^{2} & =\frac{1}{d^{3}}+\frac{1}{2 d^{2}} A+\frac{1}{4 d} B+\frac{1}{8} C=\frac{1}{d^{3}}+\frac{1}{2 d^{2}} A+\frac{1}{d}\left[\left(\frac{1}{2}-\frac{1}{d}\right) A+\frac{3}{d}-\frac{3}{d^{2}}\right]+\frac{1}{8} C \\
& =\frac{3}{d^{2}}-\frac{2}{d^{3}}+\left(\frac{1}{d}-\frac{1}{d^{2}}\right) A+\frac{1}{8} C .
\end{aligned}
$$

Thus we get

$$
\begin{aligned}
\operatorname{tr} \rho^{2}-\operatorname{tr} \rho_{1}^{2} & =\left(\frac{3}{d^{2}}-\frac{2}{d^{3}}-\frac{1}{d}\right)+\frac{2 d-2-d^{2}}{2 d^{2}}\left\|T^{(1)}\right\|^{2}+\left(\frac{1}{d}-\frac{1}{d^{2}}\right)\left(\left\|T^{(2)}\right\|^{2}+\left\|T^{(3)}\right\|^{2}\right)+\frac{1}{8} C \\
& \geq \frac{-d^{2}+3 d-2}{d^{3}}+\frac{-d^{2}+2 d-2}{2 d^{2}} \frac{2(d-1)}{d}+\frac{1}{8} C=\frac{1}{8} C-\frac{(d-1)^{2}}{d^{2}}
\end{aligned}
$$

where we have used $\left\|T^{(1)}\right\|^{2} \leq \frac{2(d-1)}{d}$ and set $\left(\left.\left\|T^{(2)}\right\|\right|^{2}+\left\|T^{(3)}\right\|^{2}\right)=0$ to get the inequality.

Similarly, one obtains

$$
\operatorname{tr} \rho^{2}-\operatorname{tr} \rho_{2}^{2} \geq \frac{1}{8} C-\frac{(d-1)^{2}}{d^{2}}, \quad \operatorname{tr} \rho^{2}-\operatorname{tr} \rho_{3}^{2} \geq \frac{1}{8} C-\frac{(d-1)^{2}}{d^{2}}
$$

Thus we have

$$
\begin{aligned}
C_{G M E}^{2}(|\psi\rangle) & =\min \left\{1-\operatorname{tr} \rho_{1}^{2}, 1-\operatorname{tr} \rho_{2}^{2}, 1-\operatorname{tr} \rho_{3}^{2}\right\}=\min \left\{\operatorname{tr} \rho^{2}-\operatorname{tr} \rho_{1}^{2}, \operatorname{tr} \rho^{2}-\operatorname{tr} \rho_{2}^{2}, \operatorname{tr} \rho^{2}-\operatorname{tr} \rho_{3}^{2}\right\} \\
& \geq \max \left\{\frac{1}{8} C-\frac{(d-1)^{2}}{d^{2}}, 0\right\}
\end{aligned}
$$

We now consider mixed quantum state $\rho \in H_{1}^{d} \otimes H_{2}^{d} \otimes H_{3}^{d}$. Let $\rho=\sum p_{\alpha}\left|\psi_{\alpha}\right\rangle\left\langle\psi_{\alpha}\right|$ be the optimal ensemble decomposition of $\rho$. We obtain

$$
C_{G M E}(\rho)=\sum p_{\alpha} C_{G M E}\left(\left|\psi_{\alpha}\right\rangle\right) \geq \max \left\{\left(\frac{1}{2 \sqrt{2}}\left\|T_{\alpha}^{(123)}\right\|-\frac{(d-1)}{d}\right), 0\right\} \sum p_{\alpha}=\max \left\{\frac{1}{2 \sqrt{2}}\left\|T^{(123)}\right\|-\frac{(d-1)}{d}, 0\right\},
$$

where we have used $\sqrt{a-b} \geq \sqrt{a}-\sqrt{b}$ for $a>b>0$ and the convex property of the Frobenius norm.

Example 2: We consider the mixture of the GHZ state and $\mathrm{W}$ state in three-qubit quantum systems $\rho=$ $\frac{1-x-y}{8} I+x|G H Z\rangle\langle G H Z|+y| W\rangle\langle W|$, where $|G H Z\rangle=$ $\frac{1}{\sqrt{2}}(|000\rangle+|111\rangle)$ and $|W\rangle=\frac{1}{\sqrt{3}}(|001\rangle+|010\rangle+|100\rangle)$. The lower bound of GME concurrence for $\rho$ by Theorem 2 is computed to be $\max \left\{\frac{1}{12}\left(\sqrt{72 x^{2}+66 y^{2}}-6\right), 0\right\}$ as shown in Figure 2. Comparing with Vicente's criterion, our lower bound can detect more genuine multipartite entangled states as shown in Figure 3.

Example 3: We consider a mixed state in threequtrit quantum systems $\rho=\frac{1-x}{27} I+x|\psi\rangle\langle\psi|$, where $|\psi\rangle=\frac{1}{\sqrt{3}}(|012\rangle+|021\rangle+|111\rangle)$. The lower bound of GME concurrence can detect GME better than Vicente's criterion(Theorem 1) and Theorem 1 in this manuscript as shown in Figure 4.

\section{CONCLUSIONS AND DISCUSSIONS}

It is a basic and fundamental question in quantum theory to detect and quantify GME. Since the GME concurrence is defined by optimization over all ensemble decom- 


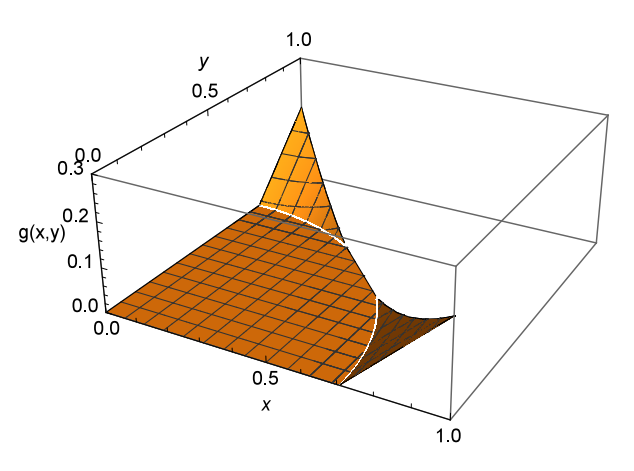

FIG. 2: Lower bound of GME concurrence in Theorem 2 for $\rho$ in Example 2. $g(x, y)$ stands for the lower bound.

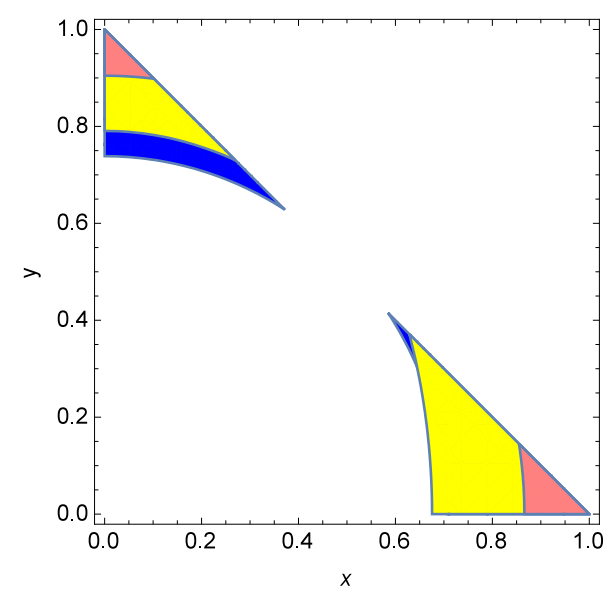

FIG. 3: GME Detected by vicente criterion (pink region by Theorem 1 and yellow region by Theorem 2 in [19]) and by the lower bound for GME concurrence in our Theorem 2 (blue region).

positions of a mixed quantum state, it is a formidable task to derive an analytical formula. We have derived an analytical and experimentally feasible lower bound for GME concurrence of any tripartite quantum state based on the correlation tensors of the density matrix. We have also obtained an effective criterion to detecting GME for any tripartite quantum states by sketching the Vicente's method. Genuine multipartite entanglement can be detected by using this bound. The results presented in this manuscript are experimentally feasible as the elements in the correlation tensors are just the mean values of the Hermitian $S U(d)$ generators. The approach used in this manuscript can also be implemented to investigate the k-separability of multipartite quantum systems. The results can be also generalized to any multipartite qudit systems.

Acknowledgments This work is supported by the NSFC No.11775306, No.11701568, No.11675113; the Fundamental Research Funds for the Central Universi-

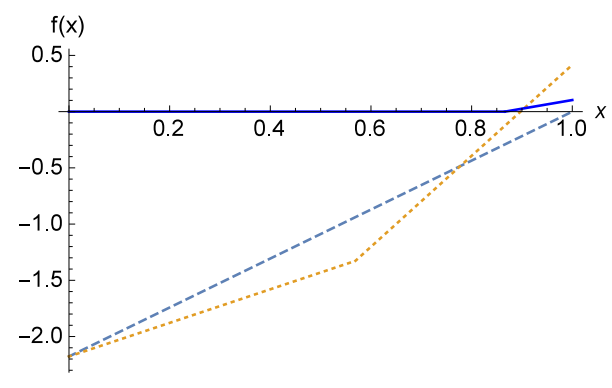

FIG. 4: GME detection: dashed line by Theorem 1 in [19]), dotted and solid line by our Theorem 1 and Theorem 2 respectively. $f(x)$ denotes the difference between the left and the right side of the inequalities in these criteria. We compute that $f(x)=2.17732(-1+x),-3.628874+4.044882 x$ and $\max \{0.222222(-3+3.4641 x), 0\}$ for Theorem 1 in [19], our Theorem 1 and Theorem 2 respectively. One finds from the figure that Theorem 1 in 19] can not detect GME for the whole region of $x$ in this example, Theorem 2 in [19] can not be operated on this states since it only fits for tripartite qubits system, while Theorem 1 and Theorem 2 in this manuscript detect GME for $0.9<x \leq 1$ and $0.866025<x \leq 1$ respectively.

ties Grants No. 15CX05062A, No. 16CX02049A, and 17CX02033A; the Shandong Provincial Natural Science Foundation No.ZR2016AQ06 and No. ZR2017BA019; Qingdao applied basic research program No. 15-9-1-103jch, the NSF of China under Grant No. 11675113, and a project sponsored by SRF for ROCS, SEM.

[1] M.A. Nielsen and I.L. Chuang, Quantum Computation and Quantum Information. Cambridge: Cambridge University Press, (2000).

[2] See, for example, D.P. Di Vincenzo, Science 270,255 (1995).

[3] O. Guhne, and G. Toth, Physics Reports 474, 1 (2009).

[4] R. Horodecki et al., Rev. Mod. Phys. 81, 865 (2009).

[5] H.J. Briegel, D.E. Browne, W. Dür, R. Raussendorf and M. Van den Nest, Nat. Phys. 5, 19 (2009).

[6] A. Sen(De) and U. Sen, Phys. News 40, 17 (2010). arXiv:1105.2412

[7] N. Gisin, G. Ribordy, W. Tittel, and H. Zbinden, Rev. Mod. Phy. 74, 145 (2002).

[8] R. Cleve et al., Phys. Rev. Lett. 83, 648 (1999); A. Karlsson et al., Phys. Rev. A 59, 162 (1999).

[9] A. S. Srensen and K. Mlmer, Phys. Rev. Lett. 86, 4431 (2001).

[10] P. Hyllus, W. Laskowski, R. Krischek, C. Schwemmer, W. Wieczorek, H. Weinfurter, L. PezzAe, and A. Smerzi, Phys. Rev. A 85, 022321 (2012).

[11] R. Raussendorf and H.J. Briegel, Phys. Rev. Lett 86, 5188 (2001).

[12] M. Murao, D. Jonathan, M.B. Plenio and V. Vedral, Phys. Rev. A 59, 156-161 (1999).

[13] M. Hillery, V. Buzek, A. Berthiaume, Phys. Rev. A. 59, 
1829 (1999).

[14] V. Scarani and N. Gisin, Phys. Rev. Lett. 87, 117901 (2001).

[15] Z. Zhao, Y.A. Chen, A.N. Zhang, T. Yang, H.J. Briegel and J.W. Pan, Nature 430, 54 (2004).

[16] Y. Yeo and W.K. Chua, Phys. Rev. Lett. 96, 060502 (2006), P.X. Chen, S.Y. Zhu, and G.C. Guo, Phys. Rev. A 74,032324 (2006).

[17] M. Huber, F. Mintert, A. Gabriel, and B. C. Hiesmayr, Phys. Rev. Lett. 104, 210501 (2010).

[18] M. Huber and R. Sengupta, Phys. Rev. Lett. 113, 100501 (2014).

[19] J.I. de Vicente, M. Huber, Phys. Rev. A, 84, 062306 (2011).

[20] J.Y. Wu, H. Kampermann, D. Bruß, C. Klockl, and M. Huber, Phys. Rev. A 86, 022319 (2012).

[21] M. Huber, M. Perarnau-Llobet, J.I. de Vicente, Phys. Rev. A 88, 042328 (2013).

[22] J. Sperling, W. Vogel, Phys. Rev. Lett. 111, 110503 (2013).

[23] C. Eltschka and J. Siewert, Phys. Rev. Lett. 108, 020502 (2012).

[24] B. Jungnitsch, T. Moroder, and O. Gühne, Phys. Rev. Lett. 106, 190502 (2011).

[25] V. Coffman, J. Kundu, and W. K. Wootters, Phys. Rev. A 61, 052306 (2000); T.J. Osborne and F. Verstraete, Phys. Rev. Lett. 96, 220503 (2006); B. Regula, S.D. Martino, S. Lee, and G. Adesso, Phys. Rev. Lett. 113, 110501 (2014).

[26] C. Klckl, M. Huber, Phys. Rev. A 91, 042339 (2015).

[27] M. Markiewicz, W. Laskowski, T. Paterek, and M. Żukowski Phys. Rev. A 87, 034301 (2013).

[28] Z.H. Ma, Z.H. Chen, J.L. Chen, C. Spengler, A. Gabriel, and M. Huber, Phys. Rev. A 83, 062325 (2011).
[29] Z.H. Chen, Z.H. Ma, J.L. Chen, and S. Severini, Phys. Rev. A 85, 062320 (2012).

[30] Y. Hong, T. Gao, and F.L. Yan, Phys. Rev. A 86, 062323 (2012).

[31] T. Gao,. F.L. Yan, and S.J. van Enk, Phys. Rev. Lett. 112, 180501 (2014).

[32] J.D. Bancal, N. Gisin, Y.C. Liang, and S. Pironio, Phys. Rev. Lett. 106, 250404 (2011).

[33] C. Lancien, O. Guhne, R. Sengupta, M. Huber, J. Phys. A: Math. Theor. 48505302 (2015).

[34] W.K. Wootters, Phys. Rev. Lett. 80, 2245 (1998).

[35] L. Aolita and F. Mintert, Phys. Rev. Lett. 97, 050501 (2006).

[36] A.R.R. Carvalho, F. Mintert, and A. Buchleitner, Phys. Rev. Lett. 93, 230501 (2004).

[37] K. Chen, S. Albeverio and S.M. Fei, Phys. Rev. Lett. 95, 040504 (2005).

[38] J.I. de Vicente, Quantum Inf. Comput. 7, 624(2007).

[39] J.I. de Vicente, J. Phys. A: Math. and Theor., 41, 065309 (2008).

[40] A.S. M. Hassan, P. S. Joag, Quant. Inf. Comput. 8, 0773 (2008).

[41] M. Li, J. Wang, S.M. Fei and X.Q. Li-Jost, Phys. Rev. A, 89,022325 (2014).

[42] A.S. M. Hassan, P. S. Joag, Phys. Rev. A, 77, 062334 (2008).

[43] A.S. M. Hassan, P. S. Joag, Phys. Rev. A, 80, 042302 (2009).

[44] M. Li and S.M. Fei, Phys. Rev. A, 86, 052119 (2012).

[45] M. Li, J. Wang, S.M. Fei, X.Q. Li-Jost, and H. Fan, Phys. Rev. A 92, 062338 (2015).

[46] G. Kimura, Phys. Lett. A, 314, 339(2003). 